\title{
Estudio Delphi de las competencias pedagógicas del profesional de Enfermería en la escuela
}

\author{
Raquel Ayuso Margañón³, Mª Cruz Molina Garúz², José Luis Medina Moya³ \\ 'Doctora en Educación y Sociedad. Grado de Educación Primaria. Licenciada en Humanidades. Diplomada en Enfermería. Instituto. \\ Catalán de la Salud. Profesora asociada. Departamento de Enfermería de Salud Pública, Salud Mental y Materno-Infantil. Facultad \\ de Medicina y Ciencias de la Salud. Universidad de Barcelona. Barcelona \\ ¿Doctora en Medicina. Máster en Promoción y Educación para la salud. Profesora titular. Departamento de Métodos de \\ Investigación y Diagnóstico en Educación. Facultad de Educación. Universidad de Barcelona. Barcelona \\ ${ }^{3}$ Doctor en Ciencias de la Educación. Licenciado en Pedagogía. Diplomado en Enfermería. Profesor titular. Departamento de \\ Didáctica y Organización Educativa. Facultad de Educación. Universidad de Barcelona. Barcelona
}

Fecha de recepción: 13 de septiembre de 2018. Aceptada su publicación: 7 de mayo de 2019.

\section{Resumen}

Objetivo: definir al profesional de Enfermería promotor de salud en el entorno escolar (PEPSEE), así como su perfil competencial pedagógico: actitudes, habilidades y conocimientos necesarios para llevar a cabo una acción de calidad y efectiva en dicho entorno. Método: se planteó una investigación de tipo cualitativa, de corte interpretativo y diseño mixto. En la recogida de datos se combinó la revisión documental con el método Delphi como técnica basada en cuestionarios a expertos, cuyo desarrollo quedó establecido en tres rondas. Hubo diferentes momentos de análisis en función de la tipología de los cuestionarios que se aplicaron, combinándose el análisis de contenido con el análisis estadístico descriptivo. Por último, se efectuó un proceso de relación y comparación de los datos obtenidos, procediendo al redactado de los resultados.

Resultados: se extrajeron 11 rasgos definitorios para el PEPSEE delimitados en tres categorías: rol educador, visión integral del educando y enfoque integrador de la acción, y 81 elementos competenciales delimitados en nueve categorías: conocimiento base para la enseñanza de salud en la escuela, competencia interpersonal y de trabajo en equipo, habilidades de mediación pedagógica de salud, comunicación educativa, actitud docente competente, valores profesionales inherentes a la dimensión ética, investigación pedagógica en promoción de salud, competencia digital e innovación pedagógica, coordinación y liderazgo educativo.

Conclusiones: el profesional de Enfermería promotor de salud en el entorno escolar es un profesional con identidad propia que debe ser reconocido social y profesionalmente como garante de una formación en salud de calidad.

\section{Palabras clave}

Promoción de la salud; educación en salud; Enfermería escolar; promoción de salud escolar; competencia profesional.

\section{Abstract \\ Delphi Study about the educational skills by the School Nursing Professional}

Objective: to define the Nursing professional as promoter of health in the school setting (NPPHSS), as well as their profile of educational skills: attitudes, skills and knowledge required to conduct a quality action which is effective in said setting.

Method: a qualitative research was conducted, with interpretive and mixed design. Data collection combined documentary review with the Delphi method as a technique based on questionnaires to experts, with its development established in three rounds. There were different moments of analysis based on the type of questionnaires applied: content analysis was combined with descriptive statistical analysis. Finally, there was a process of association and comparison of the data collected, and the results were then written down.

Results: eleven (11) defining features were obtained for the NPPHSS, classified into three categories: educational role, comprehensive vision of the learner, and integrative approach to action; and 81 competence elements classified into nine categories: basic knowledge for health education at school, interpersonal and teamwork skills, educational communication, skills for pedagogical mediation in health, competent teaching attitude, professional values inherent to the ethics dimension, pedagogical research in health promotion, digital skills and pedagogical innovation, educational coordination and leadership). Conclusions: nursing professionals as health promoters in the school setting are professionals with their own identity, who must be social and professionally acknowledged as guarantors for goodquality health training.

\section{Key words}

Health promotion; health education; school nursing; promotion of school health; professional skills.

\section{Introducción}

Por sus conocimientos, el profesional de Enfermería es idóneo para llevar a cabo educación para la salud (EpS). De hecho, sustenta el liderazgo de la EpS dirigida hacia los pacientes con enfermedades crónicas, estableciéndose como guía principal en el autocuidado $(1,2)$. Pero no solo es importante ofrecer contenidos de salud, sino que es imprescindible poseer los valores pedagógicos

\section{Dirección de contacto:}

Raquel Ayuso Margañón. CAP Riu Nord-Riu Sud.C/ Major, 49-53. 08921 Sta. Coloma de Gramenet (Barcelona). Email: raquelayusom@ub.edu

\section{Cómo citar este artículo:}

Ayuso Margañón R, Molina Garúz MC, Medina Moya JL. Estudio Delphi de las competencias pedagógicas del profesional de Enfermería en la escuela. Metas Enferm jun 201 9; 22(5):49-58. 
necesarios para realizar dicha acción de forma efectiva $(3,4)$.

Desde esta perspectiva, en el papel que juega la profesión enfermera para el desarrollo de la EpS en el entorno escolar surgen diferentes cuestiones a investigar, como son si puede una enfermera generalista cubrir esta función, si debe poseer dominio en el área de Enfermería Comunitaria, si ha de pertenecer al área de pediatría, o qué competencias pedagógicas debería tener. Es aquí donde se podría considerar que existe un factor determinante en el tipo de educación que se brinda a la comunidad. Los requisitos para desarrollar una intervención de calidad en promoción de salud implican disponer de recursos apropiados, formación del personal y una cultura de mejora de la calidad (5). En la Quinta Conferencia Mundial de Promoción de la Salud (PS) se instaba a desarrollar una masa crítica de profesionales competentes destacando la importancia de fortalecer la PS en los programas de formación. La capacitación se convierte así en un condicionante para lograr un sistema sanitario orientado a la prevención y promoción de la salud (6).

Las investigaciones realizadas sobre las necesidades de formación de profesionales en Promoción de la Salud apuntan en parte al escaso conocimiento sobre el campo teórico de la misma. Un factor fundamental ha sido la escasez de la formación de pregrado en estos temas, así como la falta de formación postgrado que la palíe (7). El informe SESPAS 2000, en el que se analizaron 131 planes de estudios en la educación universitaria española, puso de manifiesto que la PS tenía una importancia curricular deficiente en las titulaciones de ciencias de la salud (8,9). De esto se deduce que muchos de los profesionales de Enfermería en activo que actúan como agentes promotores de salud no han recibido formación básica en esta materia.

Diversos autores ponen de manifiesto la necesidad de potenciar la formación en PS en estudiantes de Enfermería, con objeto de lograr una mejor disposición para motivar a las personas a adoptar comportamientos saludables y colaborar con ellas en el cambio de los aspectos sociales perjudiciales (10-12). Así, para el desarrollo de la PS y la práctica de la EpS, es condición necesaria disponer de una formación y desarrollar unas competencias pedagógicas coherentes con el papel de los profesionales. Este aspecto ha conformado el problema principal que ha guiado la investigación, cuyo objetivo ha sido definir al profesional de Enfermería promotor de salud en el entorno escolar (PEPSEE) y describir su perfil competencial pedagógico: actitudes, habilidades y conocimientos necesarios para llevar a cabo una acción de calidad y efectiva en dicho entorno.

\section{Método}

Se efectuó un diseño mixto de investigación, con un predominio del abordaje cualitativo y de corte interpretativo a través del método Delphi. Se optó por este procedimiento para alcanzar una definición consensuada del PEPSEE a través de un panel de expertos, así como la consolidación del mapa de competencias pedagógicas (13). Se siguió una estrategia secuencial con estatus dominante de uno de los dos métodos en función del interés surgido en cada fase, con integración de datos en la interpretación y cuyo énfasis fue interpretar relaciones para obtener mayor riqueza en los resultados y mejor entendimiento del fenómeno estudiado $(14,15)$.

Para aplicar el método se siguió la propuesta planteada por Landeta (16). Primeramente se procedió a la selección de los expertos mediante un muestreo teórico inicial teniendo en cuenta los criterios definidos por Morse, priorizando la capacidad de los sujetos cuyo conocimiento y experiencia pudieran aportar reflexiones interesantes y estuvieran interesados en participar (17). Para determinar su representatividad se aplicó el denominado procedimiento Biograma, estableciendo como criterios de inclusión (18): el grado de conocimientos en la promoción de la salud en la escuela, la formación académica y científica (investigaciones o acciones formativas relacionadas con la PS en la escuela) y el grado de especialización sobre el objeto de estudio.

No hay forma de determinar cuál es el número óptimo de expertos en un panel. Según Landeta (16) el que más se repite en las investigaciones no es inferior a siete, por lo que se estableció esta cifra como un mínimo teórico. Para su reclutamiento se efectuó el mapeo asistiendo a congresos relacionados con el objeto de investigación. Esto permitió situar el contexto en el que interactuaban, obteniendo así un cuadro completo de los rasgos más relevantes del fenómeno de estudio. El panel quedó formado por 18 expertos procedentes de Madrid, Barcelona y Andorra. En la Tabla 1 se pueden observar las características de los expertos que participaron en la consulta.

También se tuvieron en cuenta el número y extensión de las preguntas, poniendo especial énfasis en su clari- 


\begin{tabular}{|c|c|}
\hline Perfil & Descripción \\
\hline Formación académica & $\begin{array}{l}\text { Trece titulados en Enfermería (diploma y/o grado), tres licenciados en pedagogía y/o ciencias de } \\
\text { la educación, y dos con grado de doctor. La totalidad del grupo disponía de una amplia formación } \\
\text { postgrado en el área de Enfermería y salud escolar, Enfermería pediátrica, promoción de salud } \\
\text { y salud pública, investigación, dirección y gestión de centros educativos, bioética y nuevas } \\
\text { tecnologías de la educación, entre otros, habiendo asistido y participado en cursos, congresos y/o } \\
\text { jornadas de carácter nacional o internacional relacionados con el objeto de estudio }\end{array}$ \\
\hline Actividades de carácter científico & $\begin{array}{l}\text { Publicaciones en revistas científicas, libros, boletines informativos, periódicos, entre otros } \\
\text { Participación en comités, sociedades científicas, grupos de investigación }\end{array}$ \\
\hline Docencia & $\begin{array}{l}\text { Más de la mitad del grupo ha realizado docencia universitaria en pregrado, postgrado y/o máster } \\
\text { Participación en otras actividades relacionadas con la docencia y/o innovación docente }\end{array}$ \\
\hline Experiencia profesional & $\begin{array}{l}\text { Amplia experiencia profesional en: } \\
\text { - Proyectos relacionados con la salud y promoción de la salud, dirigidos a estudiantes y/o formadores } \\
\text { - Ejercicio de enfermera en centros escolares (primaria, secundaria, ciclos formativos, bachillerato, } \\
\text { educación especial), así como en el ámbito comunitario y/o pediátrico } \\
\text { - Creación, gestión y/o coordinación de servicios y proyectos de Enfermería y salud escolar } \\
\text { - Referencia de programas de salud escolar (Salut i Escola, entre otros) } \\
\text { - Coordinación de servicios educativos }\end{array}$ \\
\hline
\end{tabular}

dad y extensión; el feedback cualitativo y cuantitativo al final de cada ronda, devolviendo un análisis de las respuestas y manteniendo el interés por la investigación; y el anonimato en la identidad de los expertos y sus respuestas. Por cada ronda fue preciso hacer un piloto con un grupo de terceros, ajenos a la investigación. El envío de los cuestionarios definitivo se llevó a cabo entre noviembre de 2016 y junio de 2017, a través de la plataforma "Online Encuesta" (ENUVO). Dicha interfaz fue seleccionada por su funcionalidad, accesibilidad, diseño de cuestionarios, tratamiento de los datos y garantías de seguridad.

Hubo diferentes momentos de análisis en función de la tipología de cuestionarios que se aplicaron para cada ronda, combinándose el análisis de contenido con el análisis estadístico descriptivo. En la Figura 1 se especifican las etapas del proceso de investigación realizado.

\section{Primera ronda}

El cuestionario constaba de dos preguntas abiertas sobre el objeto de estudio: 1) Defina lo que en su opinión es un profesional de Enfermería promotor de salud en el entorno escolar, 2) ¿Cuáles cree que son las com- petencias pedagógicas necesarias del profesional de Enfermería para realizar la promoción de la salud en el entorno escolar? En el análisis de esta ronda se hizo una comparación sucesiva y tabulación de los datos obtenidos por el grupo con la información previa de la literatura científica. Se introdujeron adaptaciones y se generó un conjunto preliminar de rasgos definitorios y de competencias para lograr su intervención efectiva, distribuidas en nueve categorías competenciales.

\section{Segunda ronda}

El cuestionario quedó dividido en dos bloques de preguntas de respuesta cerrada referentes a los rasgos definitorios del PEPSEE y las competencias pedagógicas preestablecidas en la primera ronda, respectivamente. Se utilizó una tipología de preguntas que permitía integrar de forma numérica las respuestas. Se solicitó a los expertos una valoración de los ítems, dando puntuaciones de acuerdo con una escala Likert, en la que 0 era "No sabe/No contesta (NS/NC)", 1 era "Nada relevante", 2 "Poco relevante", 3 "Relevante", 4 "Bastante relevante" y 5 "Muy relevante". También se incorporó en cada sección de la encuesta una pregunta de respuesta abierta permitiendo explicar los juicios y

\begin{tabular}{|c|c|c|}
\hline \multicolumn{3}{|c|}{ Figura 1. Etapas del proceso investigador mediante el uso del método Delphi } \\
\hline \multicolumn{3}{|c|}{$\begin{aligned} \text { RONDA } 1 \\
\end{aligned}$} \\
\hline Dos preguntas abiertas & $\begin{array}{c}\text { Comparación sucesiva y tabulación } \\
\text { de datos }\end{array}$ & $\begin{array}{c}\text { Rasgos definitorios/caracterización y } \\
\text { nueve categorías competenciales }\end{array}$ \\
\hline \multicolumn{3}{|c|}{$\downarrow$} \\
\hline \multicolumn{3}{|c|}{ RONDA 2} \\
\hline $\begin{array}{c}\text { Preguntas valuativas (escala Likert) } \\
\text { y preguntas abiertas }\end{array}$ & $\begin{array}{l}\text { Medidas de tendencia central } \\
\text { y dispersión }\end{array}$ & Jerarquización ordenada de los datos \\
\hline \multicolumn{3}{|c|}{$\downarrow$} \\
\hline \multicolumn{3}{|c|}{ RONDA 3} \\
\hline $\begin{array}{c}\text { Preguntas valuativas (escala Likert) } \\
\text { y preguntas abiertas }\end{array}$ & $\begin{array}{c}\text { Medidas de tendencia central } \\
\text { y dispersión }\end{array}$ & Finalización del proceso \\
\hline
\end{tabular}


plantear nuevos ítems/discrepancias, ya que los estudios mediante método Delphi en los que los expertos dan sus razonamientos son significativamente más precisos (16,19). Por último, se incluyó una pregunta adicional por cada bloque para determinar el grado de conformidad de los expertos con los ítems respecto de su opinión expresada en la ronda anterior, logrando una mayor consistencia del método y validez de la respuesta grupal. Con esta segunda ronda se consiguió una jerarquización ordenada de los ítems sobre los que se preguntaba a los expertos, atendiendo a la puntuación dada.

El análisis se realizó en función de tres indicadores: el grado de acuerdo en lo que respecta a la relevancia de cada ítem; el nivel de consenso alcanzado en la respuesta grupal; y el grado de conformidad de los expertos con los ítems, respecto de su opinión expresada en la ronda anterior (16). Se llevaron a cabo procedimientos estadísticos atendiendo al tipo de preguntas realizadas, utilizándose medidas de tendencia central (media, mediana y moda) y de dispersión (coeficiente de variación) para las preguntas formuladas usando la escala Likert. Esto permitió conocer los elementos mejor valorados por el grupo y con qué puntuación se obtuvo esta valoración. A partir de aquí se establecieron los criterios de acuerdo en lo que respecta a la relevancia de cada ítem y los niveles de consenso sobre la opinión del grupo.

Para establecer dichos criterios se procedió a la asignación de un valor representativo para el conjunto de los ítems a partir del cual se fijó el rango de acuerdo y consenso, quedando clasificado en bajo, moderado o alto. Para ello se calculó el recorrido de la distribución con los valores extremos que tomó la media y el coeficiente de variación, a través de la diferencia entre el valor mínimo y el valor máximo. La cifra obtenida se dividió entre el número de intervalos que se buscaba formar (tres en este caso), logrando como resultado la amplitud de cada uno de los intervalos.

Para determinar el grado de conformidad por parte del grupo, previamente definida la escala de respuestas en cinco opciones (totalmente en desacuerdo, en desacuerdo, ni de acuerdo ni en desacuerdo, de acuerdo, totalmente de acuerdo), se optó por fijar como criterio único que dos de las opciones de respuesta "de acuerdo" y "totalmente de acuerdo" recibieran al menos el 75\% de las contestaciones. Así, cuando esto sucedió, el grado de conformidad se consideró alto. Cualquier otro resultado se consideró bajo grado de aprobación, lo que llevó a pensar que los ítems no habían sido bien entendidos o no habían expresado completamente lo que el grupo quiso opinar en la primera ronda, por lo que requirió de reformulación para la tercera iteración. En la Tabla 2 se reflejan estos criterios detalladamente.

\section{Tercera ronda}

Se pidió a los expertos una nueva valoración siguiendo el criterio de importancia de los ítems extraídos en la anterior ronda, quedando distribuido el cuestionario en idénticas partes.

El análisis se realizó siguiendo los indicadores de acuerdo y consenso fijados en la segunda ronda manteniéndose los mismos criterios. En aquellos casos de moderado consenso en los cuales era preciso tomar una decisión se añadió una regla para considerar dicha decisión suficiente, que consistió en que el porcentaje de respuestas situadas en el intervalo definido por la mediana \pm 1 fuera superior al $80 \%$ (Tabla 3).

A medida que los diferentes ítems obtuvieron valores altos de consenso y acuerdo se fueron conformando las competencias pedagógicas atribuibles a los PEPSEE. Por el contrario, cuando el grado de acuerdo y consenso

\begin{tabular}{cccccc}
\hline $\begin{array}{c}\text { Tabla 2. Criterios de acuerdo y consenso en la respuesta grupal. Segunda ronda } \\
\text { Acuerdo } \\
\text { Medias }\end{array}$ & $\begin{array}{c}\text { Consenso } \\
\text { Coeficiente de variación }\end{array}$ & Conformidad & \\
\hline $3,71-4,11$ & Bajo & $0,23-0,32$ & Bajo & $\begin{array}{c}\text { Porcentaje de respuestas "de acuerdo" } \\
\text { "totalmente de acuerdo" } \geq 75 \%\end{array}$ & Alto \\
\hline $4,12-4,51$ & Moderado & $0,14-0,22$ & Moderado & Cualquier otro resultado & Bajo \\
\hline $4,52-4,93$ & Alto & $0,05-0,13$ & Alto & & \\
\hline
\end{tabular}

Tabla 3. Criterios de acuerdo y consenso en la respuesta grupal. Tercera ronda

\begin{tabular}{ccccc} 
& $\begin{array}{c}\text { Acuerdo } \\
\text { Medias }\end{array}$ & \multicolumn{2}{c}{$\begin{array}{c}\text { Consenso } \\
\text { Coeficiente de variación }\end{array}$} & Confirmación consenso \\
\hline $3,67-4,11$ & Bajo & $0,23-0,32$ & Bajo & Mediana $\pm 1<80 \%$ (NO) \\
\hline $4,12-4,55$ & Moderado & $0,12-0,22$ & Moderado & Mediana $\pm 1 \geq 80 \%$ (Sí) \\
\hline $4,56-5,00$ & Alto & $0,00-0,11$ & Alto & \\
\hline
\end{tabular}


fue bajo para el ítem en cuestión, dicho elemento fue eliminado del listado definitivo. Aquellos ítems que alcanzaron un acuerdo y/o consenso moderado requirieron de análisis individualizado para su integración y posición en el ranking. El manejo de los códigos cualitativos se hizo con el programa ATLAS-Ti, lo que permitió la conversión del texto en formato cuantificable y facilitó la construcción de categorías de análisis. Para el manejo de los datos cuantitativos se utilizó el programa SPSS versión 24.

En lo que respecta a los criterios de rigor científico y las consideraciones éticas se siguieron las recomendaciones de Guba (20) sobre validez interna o credibilidad, consistencia, transferibilidad o aplicabilidad y confirmabilidad. Se facilitó a los participantes un consentimiento informado, garantizándose su anonimato y privacidad, a través de acceso restringido a la información almacenada con contraseña y garantía de destrucción de grabaciones y transcripciones una vez terminada la investigación.

Por último, se obtuvieron los permisos pertinentes según la tipología de derechos de autor de las obras utilizadas y se obtuvo el certificado favorable de la Comisión de bioética de la Universidad de Barcelona.

\section{Resultados}

La primera iteración se llevó a cabo durante los meses de octubre y noviembre de 2016. De las 18 personas invitadas, 14 decidieron participar, obteniéndose 188 respuestas en total. En la primera pregunta abierta se extrajeron 42 unidades de significado, a partir de las cuales se obtuvieron diferentes rasgos definitorios del PEPSEE, quedando organizados en tres categorías.

La mayoría se refirieron a diferentes características que esencialmente se desprenden del rol educador, aludiendo a su concepción como mediador de salud individual y colectiva; a la capacitación pedagógica específica que debe tener; y a su sensibilidad docente, capaz de asentar valores pedagógicos en el proceso de enseñanza-aprendizaje.

También se destacó la perspectiva holística del individuo y la reciprocidad entre disciplinas para desarrollar la promoción de la salud en la escuela. Estos rasgos conformaron las subcategorías de análisis. En la segunda pregunta abierta se extrajeron 106 unidades de significado. Se expuso una gran variedad de elementos competenciales, cuya interpretación llevó a un listado de

\begin{tabular}{|c|c|}
\hline \multicolumn{2}{|l|}{ DOCUMENTOS PRIMARIOS } \\
\hline Categoría P1: RONDA 1-PREGUNTA 1 & Totales \\
\hline 1. Rol educador & 27 \\
\hline 2. Enfoque integrador de la acción & 8 \\
\hline 3. Visión integral del educando & 8 \\
\hline Totales & 42 \\
\hline \multicolumn{2}{|l|}{ Categoría P1: RONDA 1-PREGUNTA 2} \\
\hline $\begin{array}{l}\text { 1. Conocimiento base para la enseñanza de salud } \\
\text { en la escuela }\end{array}$ & 25 \\
\hline 2. Competencia interpersonal & 22 \\
\hline 3. Habilidades de mediación pedagógica & 18 \\
\hline 4. Comunicación educativa & 12 \\
\hline 5. Actitud docente competente & 8 \\
\hline $\begin{array}{l}\text { 6. Valores profesionales inherentes a la dimensión } \\
\text { ética }\end{array}$ & 6 \\
\hline 7. Investigación pedagógica en promoción de salud & 6 \\
\hline 8. Competencia digital e innovación pedagógica & 6 \\
\hline 9. Coordinación y liderazgo educativo & 3 \\
\hline Totales & 106 \\
\hline
\end{tabular}

competencias provisionales específicas clasificadas en subcategorías en torno a nueve categorías (Tabla 4).

En la segunda ronda participaron 14 personas y se lograron 1.504 respuestas. Se partió de nueve rasgos definitorios y 84 elementos competenciales. Tres ítems no alcanzaron acuerdo ni consenso, 44 obtuvieron nivel moderado, 34 nivel alto, y 12 no fueron coincidentes. No se logró conformidad en la categoría de "competencia digital e innovación pedagógica", que requirió de reformulación.

En la tercera ronda permanecieron 12 expertos, obteniéndose 1.249 respuestas. Se partió de 11 rasgos definitorios y 81 elementos competenciales. Siete ítems no llegaron al nivel mínimo de acuerdo ni de consenso, 47 alcanzaron un alto nivel y 38 ítems obtuvieron puntuaciones moderadas, siendo preciso optar por otros criterios para tomar una decisión.

Tanto en la segunda como en la tercera ronda, las puntuaciones medias fueron altas con una media de coeficiente de variación (CV) de 4,52 y de 4,63, respectivamente. De esto se deduce que la mayoría de los ítems propuestos fueron acertados, teniendo una alta importancia para el grupo. Asimismo, se alcanzó un grado de consenso moderado-alto para la mayoría de los ítems, mejorando incluso en la tercera ronda con un promedio de $\mathrm{CV}=0,14$ y $C V=0,12$, respectivamente. Una vez realizada la ordenación de todos los ítems analizados en la tercera ronda se delimitó el listado concluyente de los rasgos definitorios y competencias pedagógicas del PEPSEE (Tabla 5). 
Tabla 5. Rasgos definitorios del profesional de Enfermería promotor de salud en el entorno escolar (PEPSEE) y sus competencias pedagógicas para la promoción de salud en la escuela

\section{RASGOS DEFINITORIOS DEL PEPSEE}

1. Posee conocimientos y habilidades específicas para desarrollar procesos de enseñanza-aprendizaje de salud eficaces

2. Guía y orienta al alumno en su proceso de enseñanza-aprendizaje con objeto de alcanzar el bienestar en salud

3. Utiliza metodología pedagógica apropiada y domina estrategias didácticas basadas en la evidencia científica

4. Adquiere el compromiso de educar y se concibe como parte del proceso educativo

5. Promueve el empoderamiento (que el alumno se convierta en protagonista en la construcción del propio conocimiento y adquiera responsabilidad en sus decisiones de salud en beneficio individual y comunitario)

6. Estimula acciones de salud a través de la relación interdisciplinar

7. Entiende al educando desde una perspectiva integral abarcando todos los problemas a lo largo de su escolarización

8. Posee "el saber" propio de la profesión enfermera

9. Desarrolla sensibilidad docente, valores pedagógicos más allá de la profesión enfermera

\section{COMPETENCIAS PEDAGÓGICAS DEL PEPSEE}

\section{Conocimiento base para la enseñanza de salud en la escuela}

1.1. Conocimiento de la didáctica específica de la salud

1.2. Conocimiento teórico-práctico del contenido de salud que se quiere enseñar

1.3. Conocimiento del centro escolar (organización y estructura funcional, recursos externos unidades educativas especiales)

1.4. Conocimiento del currículum educativo (finalidades, objetivos y de qué manera se integra la promoción de salud en él)

1.5. Conocimiento de la comunidad donde se lleva a cabo la enseñanza

1.6. Conocimiento de intereses personales de los alumnos en lo que respecta a su salud

1.7. Conocimiento de la estructura de la profesión docente (lenguaje propio e implicaciones en el centro escolar)

1.8. Conocimiento de las características particulares de los alumnos (capacidad y dificultades de aprendizaje)

\section{Competencia interpersonal y trabajo en equipo}

2. 1. Actitud colaborativa en el desarrollo de propuestas curriculares para el mantenimiento de la salud escolar

2.2. Capacidad de establecer diálogo pedagógico sobre intereses comunes

2.3. Habilidades sociales efectivas y actitud comunicativa de equipo

2.4. Capacidad de negociación en la resolución de problemas con el equipo y la comunidad

2.5. Participación en la planificación y operativización de acciones promotoras de salud

2.6. Capacidad de gestión de situaciones estresantes con el equipo, desarrollando inteligencia emociona

2.7. Actitud de respeto y tolerancia en la relación con el equipo y comunidad

2.8. Adaptación de los propios objetivos a los del equipo y centro

2.9. Flexibilidad en la aplicación de procedimientos según la situación educativa

\section{Habilidades de mediación pedagógica}

3.1. Seleccionar estrategias y métodos de enseñanza-aprendizaje adecuados

3.2. Utilizar materiales y recursos didácticos apropiados

3.3. Identificar elementos que pueden influir negativa o positivamente en la salud

3.4. Explorar potencialidades del alumno (conocimientos, habilidades y actitudes)

3.5. Habilidad en técnicas grupales y de fortalecimiento de grupo

3.6. Autocrítica y evaluación de la propia práctica docente

3.7. Identificar necesidades previas de aprendizaje en salud de los alumnos y de la comunidad escolar

3.8. Diseñar procesos educativos ajustados a la realidad escolar, atendiendo a las diferencias individuales y de grupo

3.9. Desarrollar situaciones de aprendizaje en salud según los conocimientos previos detectados, y establecer conexiones entre estos y los nuevos que se van adquiriendo

3.10. Conceptualizar, estructurar y secuenciar los contenidos de salud y las acciones orientadas hacia el fin educativo, adaptándose al progreso de los alumnos

3.11. Aceptar el error del alumno como parte natural del proceso de aprendizaje, dando margen a la autorregulación del mismo

3.12. Evaluación del proceso y resultado de aprendizaje del alumno de forma continua, reajustando la acción pedagógica y propiciando que este valore su propio aprendizaje

\section{Comunicación educativa}

4.1. Habilidades lingüísticas y paralingüísticas eficaces, adaptando el lenguaje propio al del alumno

4.2. Adecuar el mensaje de salud a los intereses y necesidades de los educandos

4.3. Mantener un diálogo abier to y receptivo en el aula y fuera de esta

4.4. Lenguaje aseverativo, evitando la autoridad e imposición

4.5. Acción discursiva coherente y motivadora

4.6. Usar elementos propios del lenguaje no verbal (miradas, gestos...) como instrumento transmisor de afecto, seguridad y confianza

4.7. Actitud comunicativa flexible y tolerante a la participación de los alumnos; conciliadora y armónica, basada en los acuerdos. Lenguaje afectivo 
Tabla 5. Rasgos definitorios del profesional de Enfermería promotor de salud en el entorno escolar (PEPSEE) y sus competencias pedagógicas para la promoción de salud en la escuela (cont.)

\section{COMPETENCIAS PEDAGÓGICAS DEL PEPSEE}

\section{Actitud docente competente}

5.1. Actitud tolerante y de respeto ante las diferencias en la educación

5.2. Disposición permanente al aprendizaje en la tarea pedagógica

5.3. Actitud abierta a propuestas de mejora continua en promoción de salud

5.4. Disposición a compartir ideas y aprender con los alumnos y comunidad educativa

5.5. Actitud dialógica y de negociación en la relación pedagógica

5.6. Actitud constructiva positiva hacia el aprendizaje en salud

5.7. Disposición de ayuda hacia los alumnos y el resto de la comunidad educativa

5.8. Actitud receptiva a las vivencias y realidad de los educandos

\section{Valores profesionales inherentes a la dimensión ética}

6.1. Actualización y capacitación constante en el ejercicio profesional docente

6.5. Compromiso de contribuir al bienestar escolar a través de un ejercicio docente de calidad por el que pueda ser reconocido

6.2. Disposición intrínseca y/o motivación personal por desempeñar el rol docente

6.3. Responsabilidad de desarrollar de manera óptima las intervenciones docentes de promoción de salud

6.4. Conocimiento de sí mismo tomando conciencia del rol docente

6.5. Autorreflexión sobre la propia acción pedagógica

\section{Investigación en promoción de salud}

7.1. Exploración permanente del estado de salud de la población, especialmente en la etapa escolar, así como aquellas condiciones y factores que favorecen el bienestar, evaluando el impacto de la intervención promotora en la salud del alumnado y comunidad

7.2. Actualización constante en contenidos de salud

7.3. Búsqueda constante de políticas educativas y programas eficaces de promoción de salud en la escuela

7.4. Actualización continua en docencia en función de líneas pedagógicas innovadoras

7.5. Exploración permanente de estrategias y métodos, materiales y recursos docentes renovados y adaptados al entorno escolar cambiante

\section{Competencia digital e innovación pedagógica}

8.1. Capacidad creativa y de innovación, poniendo en práctica nuevas ideas de promoción de salud a través de las TIC

8.2. Capacidad para expresarse y comunicarse con el alumnado y la comunidad (tanto de forma sincrónica como asincrónica) utilizando el lenguaje propio de las TIC

8.3. Capacidad para organizar, evaluar y optimizar los procesos educativos a través de las TIC

8.4. Dominio de las herramientas TIC y ambientes virtuales de aprendizaje en salud

8.5. Participación en redes y comunidades educativas de salud a través de las TIC. Utilización de redes sociales TEP (Facebook, Twitter...) para el posicionamiento enfermero en referencia a la promoción de salud en la escuela

8.6. Dominio y utilización de las TIC en el área investigativa

\section{Coordinación y liderazgo educativo}

9.1. Capacidad de impulsar mejoras en las condiciones de enseñanza de salud y participar de estas

9.2. Liderar el cambio en la experimentación de ideas promotoras de salud

9.3. Implicación en el desarrollo de las políticas educativas del centro respecto a la dimensión de salud

9.4. Crear las condiciones de grupo que favorezcan el aprendizaje y que aseguren la colaboración con el profesorado y la comunidad educativa, promoviendo espacios de participación

9.5. Capacidad para influir en la dirección y organización del centro en lo que respecta a la mejora de la salud, y coordinar las intervenciones consensuadas

9.6. Promover la cultura de promoción de la salud en el centro escolar

\section{Discusión y conclusiones}

Se puede afirmar que el profesional de Enfermería promotor de salud en el entorno escolar (PEPSEE) se concibe como guía y mediador activo en un proceso de enseñanza-aprendizaje intencionado de salud, transmisor de conocimientos y facilitador de las condiciones y medios necesarios que permitan convertir al alumno en el máximo responsable de su propia salud. Está comprometido y sensibilizado con la enseñanza, con valores y capacitación pedagógica específica buscando el aprendizaje en salud a lo largo de toda la escolarización. Estos resultados se avienen con lo dirimido en la literatura científica, en la cual se subraya la función encaminada a motivar y orientar como uno de los elementos más importantes del rol educador, considerando, por tanto, al profesional que lo desempeña como un mediador de los cambios del comportamiento (21). Otros autores avalan que es pertinente verse como parte del proceso, el verdadero educador es quien realmente comprende el acto pedagógico y lo asume (22).

En lo que respecta a las competencias pedagógicas del PEPSEE destaca el énfasis concedido al desarrollo de un conocimiento específico del contexto escolar y su 
estructura, currículum, alumnado y comunidad, teorías pedagógicas y metodologías de enseñanza. El conocimiento que caracterizaría al PEPSEE no es aquel que tiene cualquier profesional de Enfermería por tener un saber avanzado en salud, sino por desarrollar además un conocimiento especial para la enseñanza (23).

También se ha determinado como necesaria la colaboración activa con otros profesionales y estamentos, creando redes y alianzas en todos los aspectos que favorezcan el proceso de enseñanza-aprendizaje en salud, lo cual coincide con la propia estrategia europea de "Salud 2020" que sugiere alianzas estratégicas y movilización entre todos los sectores para lograr la esencia de la promoción de la salud en la escuela (24).

El desarrollo de habilidades de mediación pedagógica de salud ha sido una de las categorías competenciales determinantes para este estudio. Incluye habilidad para organizar acciones didácticas, escoger métodos y recursos de enseñanza adecuados que permitan alcanzar objetivos educativos de salud, teniendo en cuenta los conocimientos previos, necesidades y preferencias del educando; y desarrollar una capacidad evaluativa como parte constitutiva del proceso de enseñanza-aprendizaje. Estos resultados fueron consistentes con investigaciones anteriores que destacaron dichos aspectos en la ejecución del proceso de mediación $(25,26)$.

La acción discursiva y las habilidades sociales comunicativas adquieren especial interés para el PEPSEE. Asimismo, el desarrollo de una actitud pedagógica abierta que permita al mismo establecer una relación de confianza y respeto, que lo reafirme como profesional competente para asumir el rol docente en el entorno escolar, también ha sido consistente con la opinión de otros referentes teóricos (27-29).

El compromiso con la docencia, la motivación personal por ejercer dicho rol y la responsabilidad profesional que permite desarrollarlo de manera óptima, son los valores profesionales inherentes a la dimensión ética destacados para el PEPSEE. Se trata de llegar a reconocer las dimensiones de la acción pedagógica, de modo que el objetivo último para el enfermero no sea únicamente el cuidado de la salud de los pacientes, sino la formación de los educandos en lo que concierne al mantenimiento de esta. Tomando consciencia de su compromiso y labor profesional como educador, puede llegar a ser reconocido por la sociedad. Estos valores también han sido motivo de reflexión en la literatura científica como elementos necesarios para llevar a cabo un proceso de enseñanza-aprendizaje óptimo (30,31).

Adquirir una base de conocimiento científico en promoción de la salud y docencia constituye un elemento sustancial para validar su práctica profesional, lo que concuerda con varios autores que confirman la necesidad de aplicar la evidencia procedente de dicha investigación de forma continuada (32). La capacidad para innovar en la búsqueda de recursos y estrategias pedagógicas también ha sido considerada como necesaria, coincidiendo este resultado con otros estudios que reafirman el impacto positivo de las TIC en las respectivas áreas de actuación en el ámbito educativo (33).

Por último, ejercer un liderazgo que legitime su papel enfermero y su autoridad pedagógica, convirtiéndose en el promotor primordial para la actividad formativa en salud de la escuela. Son diversos los autores que avalan su importancia para impulsar mejoras en las condiciones de enseñanza y aprendizaje $(34,35)$.

En definitiva, el PEPSEE se concibe como un profesional capaz de ver y tratar la realidad desde diferentes perspectivas (educativa y sanitaria), pero sin perder su propia naturaleza. Podría ser una enfermera generalista, o bien enfermera pediátrica, escolar o comunitaria, siempre que sea capaz de desarrollar unas competencias pedagógicas que combinen "el saber", "el querer" y "el poder", que le otorguen una identidad propia, así como un reconocimiento social y profesional por su acción promotora de salud.

A lo largo del estudio surgieron algunas limitaciones tales como la ingente información sobre competencias desde el punto de vista educativo y laboral, que dificultó la fase analítica y síntesis de resultados. Fue difícil hallar estudios sobre el rol educador del enfermero en la escuela, estando la mayoría enfocados a la promoción de la salud en general. El tamaño de la muestra constituyó una limitación en el procedimiento analítico seleccionado. Un mayor número de participantes en el Delphi hubiera permitido hacer análisis diferenciales por grupos más precisos. Por último, la unificación de dos grandes ramas del saber (educación y sanidad) exigió un esfuerzo adicional de reflexión desde diferentes perspectivas, dificultando la relación de categorías en el proceso de análisis. A la vez constituyó una fortaleza en la riqueza de conclusiones, que pueden servir de reflexión a los referentes de salud y educación de cara 
a arbitrar estrategias para integrar a dicho profesional que garantice una formación en salud de calidad.

En conclusión, el PEPSEE en el entorno escolar es un profesional con identidad propia que debe ser reconocido social y profesionalmente como garante de una formación en salud de calidad.

\section{Financiación \\ Ninguna.}

\section{Conflicto de intereses}

Ninguno.

\section{Bibliografía}

1. Sillas D, Jordán Ml. Autocuidado, elemento esencial en la práctica de Enfermería. Práctica Clínica [internet]. 2011 [citado 8 may 2019]; 19(2):2010-2. Disponible en: http://www.index-f.com/dce/19pdf/19-067.pdf

2. Soto P, Masalan PA, Barrios S. La educación en salud, un elemento central del cuidado de Enfermería. Rev Méd Clín Las Condes [internet]. 2018 [citado 8 may 2019]; 29(3). Disponible en: https://www.sciencedirect.com/ science/article/pii/S0716864018300543

3. Peñaranda F, López J, Molina D. La educación para la salud en la salud pública: un análisis pedagógico. Hacia promoc salud. 2017; 7577(1):123-34.

4. Rozo de Arévalo C. Fundamentos pedagógicos que sustentan el proceso de educación en salud. Aquichan. 2002; 2(2):27-35.

5. Rootman I, Goodstadt M, Potvin L, Springett J. Un marco para la evaluación de la promoción de la salud. En: Evaluación de la Promoción de la Salud Principios y perspectivas. Washington: Organización Panamericana de Salud; 2007. p. 29-60.

6. Quintero E, De la Mella S, Cómez L. La promoción de la salud y su vínculo con la prevención primaria. Medicentro electrónica [internet]. 2017 [citado 8 may 2019]; 21 (2). Disponible en: http://www.medicentro.sld.cu/index.php/ medicentro/article/view/2315/1941

7. Gutiérrez J, Gallego J, Guayta R, Pérez M, Rodríguez M, Aguirre R, et al. Formación en Promoción y Educación para la Salud, Informe del Grupo de Trabajo de Promoción de la Salud a la Comisión de Salud Pública del Consejo Interterritorial del Sistema Nacional de Salud. Madrid: Ministerio de Sanidad y Consumo; 2003. p. 1-89.

8. Aibar C. Los planes de estudio: condicionantes académicos y asistenciales. En: MSSSI (ed.). Actas de la I Jornada Nacional de Formación y Educación para la Salud. Madrid: MSSSI; 2007. p. 24-7.

9. Ruano-Casado L, Ballestar-Tarín ML. Incorporación de la promoción y educación para la salud en los planes de estudio de grado en Ciencias de la Salud de la Universitat de València. Fund Educ Médica. 2015; 18(2):149-53.

10. Morín V. Percepción de los factores que condicionan la realización de actividad física en personas entre 45 y 75 años una interpretación a partir del modelo de promoción de la salud de Pender [tesis]. Universitat de Barcelona; 2014.

11. Firmino S, Mesquita Al, Costade Sousa S, De Lima C, Carvalho A, Santos M. Promoción de la salud: la calidad de vida en las prácticas de Enfermería. Enferm Glob 2013; (32):260-9

12. Grunauer G, Cortón B, Forgas M. Formación de la competencia promoción de salud en el técnico superior en Enfermería en el contexto ecuatoriano. MEDISAN [internet]. 2017 [citado 8 may 2019]; 21 (6):731-40. Disponible en: http://scielo.sld.cu/scielo.php?script=sci_arttext\&pid=\$1029-30192017000600014\&lng=es

13. Varela-Ruiz M, Díaz-Bravo L, García-Durán R. Descripción y usos del método Delphi en investigaciones del área de la salud. Investig en Educ Médica [internet]. 2012 [citado 8 may 2019];1(2):90-5. Disponible en: http:// riem.facmed.unam.mx/sites/all/archivos/V1 Num02/07_ MI_DESCRIPCION_Y_USOS.PDF

14. Creswell J, Plano V. Gutmann M, Hanson W. Advanced Mixed Methods research designs. In: Tashakkori A, Teddlie C (eds.). Handbook of Mixed Methods in social and behavioral research. 201 1. p. 209-40.

15. Cabrera L, Rosales C. Aplicación de la metodología mixta en un estudio Delphi sobre calidad educativa en enseñanza secundaria obligatoria. Campo abierto, 2017: 36(1):65-81.

16. Landeta J. El método Delphi. Una técnica de previsión del futuro. Barcelona: Ariel; 2002

17. Flick U. Introducción a la investigación cualitativa [internet]. Morata. A Coruña: Fundación Paideia Galiza; 2012 [citado 8 may 2019]. Disponible en: http://cataleg.ub. edu/record=b1689582 51*spi

18. Cabero J, Llorente Mc. La aplicación del juicio de experto como técnica de evaluación de las tecnologías de la información y comunicación (TIC). Rev Tecnol Inf y Comun en Educ. 2013; 7(2):1 1-22.

19. López-Gómez E. El método Delphi en la investigación actual en educación: una revisión teórica y metodológica. Educ XX1. 2018; 21(1):1-21

20. Guba EG. Criterios de credibilidad en la investigación naturalista. En: Gimeno J, Pérez A (eds.). La Enseñanza: su teoría y su práctica. Madrid: Akal; 1981. p. 148-65.

21. Gutiérrez F. Papel de la Enfermería en la Educación para la Salud. Complejo QPS. Rev Española Sanid Penit. 2004; 6(3).

22. Rosales MV. El docente que quiero ser: humano, sensible. Ontosemiótica Artículos Arbitr. 2014; (1):3-7.

23. Leal Castro A. El Conocimiento Didáctico del Contenido (CDC): una herramienta que contribuye en la configuración de la identidad profesional del profesor. Magistro. 2014:8(15):89-110.

24. Reglamento (UE) $n^{\circ} 282 / 2014$ del Parlamento Europeo y del Consejo, relativo a la creación de un tercer programa de acción de la Unión en el ámbito de la salud para el periodo 2014-2020 y por el que se deroga la Decisión n 1350/2. Estrasburgo: Consejo de la Unión Europa. Diario Oficial de la Unión Europea; 2014.

25. Montes de Oca N, Machado EF. Estrategias docentes y métodos de enseñanza-aprendizaje en la Educación Superior. Rev Humanidades Médicas [internet]. 2011 [citado 8 may 2019];1 1 (3). Disponible en: http://humanidadesmedicas.sld.cu/index.php/hm/article/ view/127/81

26. Villarruel M. La práctica educativa del maestro mediador Rev Iberoam Educ. 2009; 50(3):2-12.

27. Michelena N, Blanco F. Dimensión comunicativa en la Atención Primaria de salud. En: Álvarez Sintes R, Hernández Cabrera G, Báster Moro JC, García Núñez RD. Medicina Ceneral Integral. La Habana: Editorial Ciencias Médicas; 2014. p. 32-6 
28. Pacheco G, Álvarez R. Comunicación en salud y técnicas educativas. En: Álvarez Sintes R, Hernández Cabrera G, Báster Moro JC, García Núñez RD. Medicina General Integral. La Habana: Editorial Ciencias Médicas; 2014 [citado 8 may 2019]; p. 125-37.

29. Ortiz D. El constructivismo como teoría y método de enseñanza. Sophia, colección Filos la Educ. 2015; (19):93-110.

30. Rodrigo J, Imbarack P. Compromiso docente, una interpelación al sentido de la profesionalidad en tiempos de cambio. Estud Pedagógicos [internet]. 2014 [citado 8 may 2019]; XL(1):257-73. Disponible en: https://scielo. conicyt.cl/pdf/estped/v40nEspecial/art15.pdf

31. Ibarra G. El vínculo de la ética y de la identidad profesional. En: Colomer A (ed.). América Latina, Globalidad e Integración (Tommo II). Madrid: Ediciones del Orto; 2012.

32. Varela MD, Sanjurjo ML, Blanco FJ. La investiación en Enfermería. Rol de la enfermería. Enfuro. 2012; (12):1921.

33. Prieto V, Quiñones I, Ramírez G, Fuentes Z, Tahimí Labrada, Pérez O, et al. Impacto de las tecnologías de la información y las comunicaciones en la educación y nuevos paradigmas del enfoque educativo I. Educ
Médica Super [internet]. 2011 [citado 8 may 2019]; 25(1):95-102. Disponible en: http://scielo.sld.cu/pdf/ ems/v25n1/ems09111.pdf

34. Bernal A, Ibarrola S. Liderazgo del profesor: objetivo básico de la gestión educativa. Rev Iberoam Educ. 2015; 67:55-70.

35. Bolívar A. El liderazgo educativo y su papel en la mejora: una revisión actual de sus posibilidades y limitaciones. Psicoperspectivas Individuo y Soc [internet]. 2010 [citado 8 may 2019]; 9(2). Disponible en: http://www. psicoperspectivas.cl/index.php/psicoperspectivas/article/view/112/140

Si quieres ampliar más información sobre este artículo, consulta:

$\begin{array}{ll}\text { Introduce estos } & \text { Promoción de la salud escolar; educación } \\ \text { términos: } & \text { ensalud; Enfermería escolar; competen- } \\ \text { encuentr@ @ia pedagógica; método Delphi. }\end{array}$

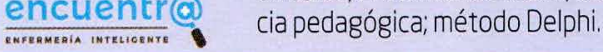


Copyright of Metas de Enfermería is the property of Difusion Avances de Enfermeria, S.L. and its content may not be copied or emailed to multiple sites or posted to a listserv without the copyright holder's express written permission. However, users may print, download, or email articles for individual use. 